\title{
Multi-label Classification of Surgical Tools with Convolutional Neural Networks
}

\author{
Jonas Prellberg \\ Dept. of Computer Science \\ University of Oldenburg \\ jonas.prellberg@uni-oldenburg.de
}

\author{
Oliver Kramer \\ Dept. of Computer Science \\ University of Oldenburg \\ oliver.kramer@uni-oldenburg.de
}

\begin{abstract}
Automatic tool detection from surgical imagery has a multitude of useful applications, such as real-time computer assistance for the surgeon. Using the successful residual network architecture, a system that can distinguish 21 different tools in cataract surgery videos is created. The videos are provided as part of the 2017 CATARACTS challenge and pose difficulties found in many real-world datasets, for example a strong class imbalance. The construction of the detection system is guided by a wide array of experiments that explore different design decisions.
\end{abstract}

Index Terms-surgical tool detection, multi-label learning, transfer learning, fine-tuning, convolutional neural networks

\section{INTRODUCTION}

Analyzing medical image data is an active area of research [1], [2] that commonly deals with radiology and histology images. However, surgeries in modern operating rooms are assisted by imaging technologies as well. For example, video recordings of surgeries allow to generate operation reports and reconstruct the surgical workflow [3] or enable computerassisted intervention systems that provide real-time recommendations and warnings to the surgeon [4]. The latter are especially useful for inexperienced surgeons or surgeons in training. Such systems should be vision-based and not rely on markers so that ordinary surgical tools can be used and the system works in any operating room [5].

Surgical tool detection in images can be used as the foundation for higher-level systems, e.g. as in [4] where surgical steps and phases are inferred from tool presence. To stimulate research in tool detection, the CATARACTS challeng $\oint^{1}$ has been organized by researchers at the Laboratory of Medical Information Processing in Brest. They provide a collection of annotated cataract surgery videos with the goal to automatically detect the presence of 21 different surgical tools in each video frame. A cataract is the clouding of the eye's natural lens and leads to poor vision. During the surgery the natural lens is destroyed and replaced with an artificial one. In machine learning terms, the challenge is a supervised classification problem on medical images.

Most previous work on surgical tool detection is performed with traditional machine learning techniques and hand-crafted features [5]. In contrast, this paper explores multiple approaches based on convolutional neural networks (CNN) to detect

\footnotetext{
${ }^{1}$ https://cataracts.grand-challenge.org
}

surgical tools in videos. CNNs have significantly improved the state of the art in vision based problems over the last years and therefore are a promising choice for this challenge. All models presented in this paper are based on the successful residual network (ResNet) [6] because it is among the best performing models in several vision challenges and implementations pretrained on ImageNet [7] are readily available in machine learning frameworks. This paper focuses on CNNs that classify single frames. While multi-frame models might improve the detection accuracy, they are vastly more difficult to train [8], which is problematic since some classes in the CATARACTS dataset have very few training examples.

In this paper a wide array of design choices is explored to create an effective CNN for surgical tool detection. First, different ways to use ResNet for transfer learning are compared. The best results are achieved by fine-tuning ResNet while keeping some layers fixed. On the other hand, using ResNet as a feature extractor performs worse than fine-tuning in all experiments. Second, the traditional CNN design for supervised learning is compared to a design proposed by Oquab et al. [9] for weakly supervised learning. As will be explained, images from the CATARACTS dataset can contain multiple objects, but bounding box information is not available. Therefore, the problem can also be cast as a weakly-supervised learning problem. However, experiments show that networks following the traditional design perform better in all cases. Third, a weighted loss function is explored to combat the class imbalance found in the dataset. This leads to slight generalization improvements. The final network achieves 0.957 AUC ${ }^{2}$ on the challenge's test set.

The remainder of this paper is organized as follows: In Section [I] related literature that was helpful for tackling this challenge is reviewed. Section III presents two families of network architectures to detect surgical tools in video frames. These networks will be used for experiments and compared against each other. Afterwards, in Section IV] the CATARACTS dataset is described. The difficulties inherent to this dataset and the approaches to solve them are explained. Finally, in Section $\mathrm{V}$, a wide array of experiments is conducted to explore possible design choices, finally resulting in the classifier model. Section VI summarizes the paper.

\footnotetext{
${ }^{2} \mathrm{AUC}$ is the area under the receiver operating characteristic (ROC) curve.
} 


\section{RELATED WORK}

Convolutional neural networks. CNNs dominate recent computer vision challenges and especially residual networks [6] are among the most successful architectures. Residual networks were the first deep architectures that did not suffer from a performance degradation due to the increased difficulty of optimizing a large number of layers. This is achieved by adding regularly spaced shortcut connections to an otherwise linear stack of layers.

Object detection. Object detection is the problem of detecting possibly multiple objects of various classes in an image and returning their locations. This is somewhat related to the problem at hand: surgical tools must be detected in an image but the location is irrelevant. However, all state-of-theart object detectors reviewed in [10] need bounding box labels for their training, which are not available in the CATARACTS dataset. Consequently, existing object detection networks are unsuitable for this problem.

Multi-label image classification. In the dataset at hand zero to three surgical tools can be visible in each video frame. Therefore, one image is associated with a set of labels instead of exactly one label. This setting is called multi-label learning. Using neural networks for multi-label problems is mostly a matter of choosing an appropriate last layer, loss function and (often implicitly) problem transformation. Specifically for CNNs, Gong et al. [11] compare different loss functions in a multi-label setting. Wei et al. [12] and Oquab et al. [9] both perform multi-label image classification by treating the task as $c$ independent binary classification problems. As such, their CNNs have $c$ output nodes which is an implicit way of applying the binary relevance transformation [13].

Transfer learning. Training CNNs with millions of weights requires a large amount of labeled training data. This is expensive or even infeasible to collect for many tasks. However, it has been shown that the lower layers of deep CNNs used for image classification learn very general filters that are applicable to different datasets as well [14]. The idea behind transfer learning is to pretrain a network on a very large image datasets, such as ImageNet. The network can then be used as a fixed feature extractor or fine-tuned by training it further on the target dataset. Both of these transfer learning approaches will be explored and compared in this paper.

Imbalanced datasets. The CATARACTS dataset is drastically imbalanced since some tools are used much more often during cataract surgery than others. This makes classification accuracy meaningless as a performance metric. Receiver operating characteristic (ROC) graphs [15] are a viable alternative that can be aggregated into a scalar performance metric by calculating the area under curve (AUC).

Furthermore, the class imbalance influences a neural network's stochastic gradient descent training. Since training examples of the majority classes are much more common, the gradient direction is dominated by examples of these majority classes [16], [17]. A possible remedy is to scale the loss by class prevalence, i.e. increasing the loss and therefore gradient length for low-prevalence classes. This approach is explored in this paper as well.

Surgical tool detection. Much of the work related to visual surgical tool detection from 2000 to 2015 has been reviewed in [5]. While the goals vary from detecting presence to estimating tool poses in 3D, all 28 reviewed methods have in common that they use hand-crafted image features as input to different modeling strategies. The same holds true for more recent publications [3], [4], [18], [19] that also work with cataract surgery datasets. Additionally, [4] employs preprocessing methods specifically tuned to the cataract surgery dataset that prevents easy application to different tool detection problems. Work using CNNs in this application domain is just beginning to emerge. EndoNet [20] is similar to this work: AlexNet is fine-tuned to detect different tools and surgical phases in laparoscopic videos. The main difference is a threetimes smaller amount of tools in their dataset. Furthermore, there is concurrent work with CNNs being done by other authors for the CATARACTS challenge.

\section{NeTWORK ARCHITECTURES}

All network architectures presented in this section build upon the 50-layer ResNet [6] because of its successes in various computer vision challenges. While larger variants of ResNet with more layers might yield improved performance, their training cost was prohibitive given the time and hardware constraints for this work. Two families of networks will be described from which several instances of networks can be derived. The first family is designed for fine-tuning experiments, while the second one uses ResNet as a fixed feature extractor. All instances are linear stacks of multiple network parts: first some layers of ResNet, then optionally some custom layers and finally the classification head. There are two choices for the classification head: $a v g-f c$ and conv-max (see Figure 1).

The $a v g-f c$ case is the standard supervised classification setup of ResNet. The feature maps created by the last convolutional layer are global average-pooled and then fed into a fully connected layer with as many units as there are classes. This approach is also found in other popular network architectures such as Inception [21] and is therefore used as the baseline in all experiments.

The conv-max case is the setup proposed by Oquab et al. [9] for weakly supervised multi-label learning. In their work AlexNet is used as a fixed feature extractor, followed by two trainable convolutional "adaption" layers and finally a global max-pooling. Because the network family here uses fine-tuning instead of a feature extractor with fixed weights, adaption layers in their original sense are unnecessary. However, one convolutional layer is still employed so that there are as many feature maps as classes before applying the global max-pooling. The paper claims that the global max-pooling "effectively searches for the best-scoring candidate object position within the image, which is crucial for weakly supervised learning where the exact position of the object within the image is not given at training" [9]. Since the CATARACTS dataset also poses a multi-label problem and has no bounding-box 


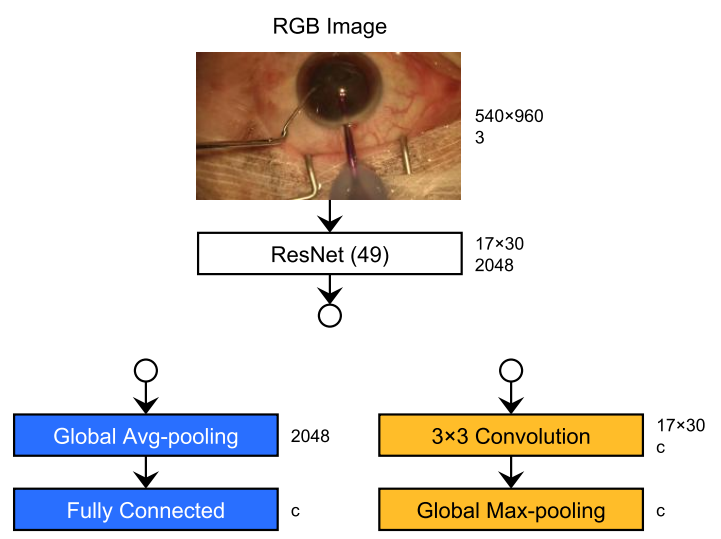

Fig. 1. Fine-tuning (FT) network family. The figure shows two possible instances. The output of the 49th ResNet layer is either fed into the avg-fc classification head (left) or the conv-max classification head (right). The labels next to each node are feature map size and feature map count. The parameter $c$ denotes the number of classes.

information, this approach seems well suited to the problem and will be compared to the traditional setup.

Both variants have $c$ outputs with $c$ being the number of unique surgical tools to detect. Also, the activation function of the last fully connected or convolutional layer respectively is the sigmoid function to squash values into the range $[0,1]$. Note that this is different from a softmax function because the $c$ outputs are independent and generally do not sum to one.

All networks presented here use transfer learning, either in the form of fine-tuning or with a fixed feature extractor. Transfer learning is essential for good performance on the CATARACTS dataset because some classes contain very few training examples. This claim will be supported empirically in Section V-B

The network family for fine-tuning (FT) experiments is illustrated in Figure 1. It uses all 49 convolutional layers of ResNet. The output feature maps of the 49th convolutional layer are either fed into the $a v g-f c$ or conv-max classification head. In general, all weights in this network family are trainable but for some experiments the first $k$ layers of ResNet will be frozen. The pretrained weights provide a form regularization that will become stronger with an increasing amount of frozen layers. In order to optimize for generalization performance, different settings are explored.

Training this architecture requires a significant amount of memory due to the many trainable weights and input image resolution. All explored instances of this fine-tuning network family vary only in the amount of ResNet layers that are frozen and the type of classification head.

The network family for fixed feature extractor (FFE) experiments is illustrated in Figure 2. The first $k$ layers of ResNet are used as a feature extractor with fixed weights. The resulting feature maps are fed to a max-pooling layer and three layers of convolutions with 384 feature maps each. Each convolution is followed by Batch Normalization [22] and a

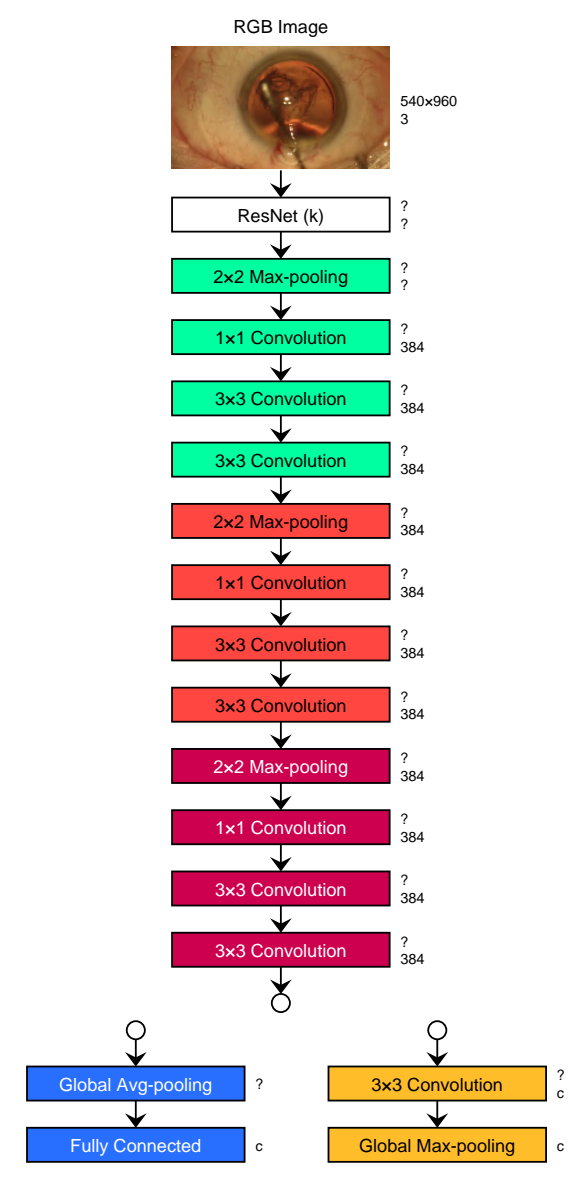

Fig. 2. Fixed feature extractor (FFE) network family. The figure shows 8 different possible instances. The input image is fed into a fixed weight ResNet with $k \in\{22,31,40,49\}$ layers. The resulting feature maps are processed by a custom architecture. Finally, the result is either fed into the $a v g$ - $f c$ classification head (bottom left) or the conv-max classification head (bottom right).

ReLU activation function. The same structure is repeated two more times. This specific custom architecture was found to be the best in preliminary experiments but is not necessarily the best possible one. Like for the first network family, the final part is either the $a v g-f c$ or conv-max classification head. Training this architecture is relatively inexpensive in terms of required memory because all ResNet weights are fixed and only the custom layers are trained. The explored instances of this family vary in the amount of ResNet layers that are used as a FFE and the type of classification head. For clarity, Figure 3 shows three specific instances of the FFE family. Note how the feature map resolution changes depending on the amount of ResNet layers used as a feature extractor.

In both network families each output node corresponds to a predicted score for one of the $c$ surgical instruments. Because the instruments are not mutually exclusive, the binary relevance transformation is used so that the task is treated as $c$ separate binary classification problems. For each of these binary problems the training dataset can be partitioned into a set of 


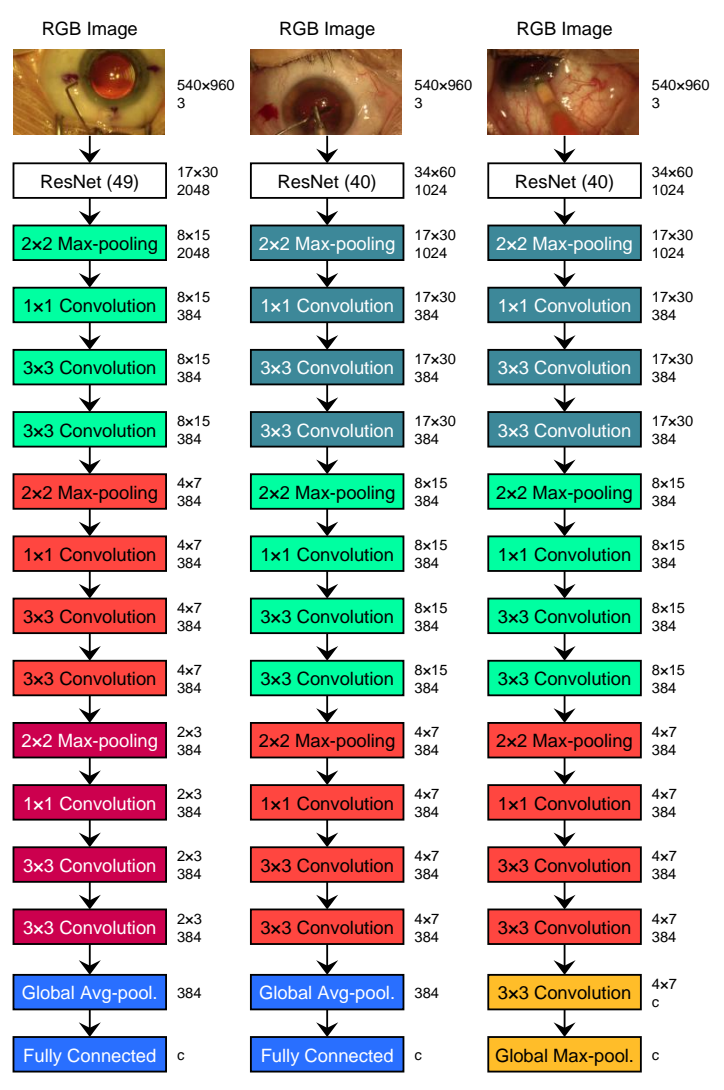

Fig. 3. Three exemplary instances of the FFE network family with different choices for $k$ and the classification head. Left: $k=49$, avg- $f c$. Center: $k=40$, avg-fc. Right: $k=40$, conv-max.

positive and negative examples. The set of positive examples for class $i$ contains all frames that show tool $i$ (potentially together with other tools), while the set of negative examples contains all other frames.

Each of the binary problems is trained using the cross-entropy loss function

$$
H(p, q)=-(1-p) \log (1-q)-p \log (q),
$$

where $p \in\{0,1\}$ is the ground-truth probability for the positive class and $q \in[0,1]$ is the predicted probability (network output) for the positive class. While the binary problems are conceptually separate, this makes no difference during training. For each example in the training set, the loss is calculated for all $c$ network outputs and then backpropagated through the network.

\section{DATASET}

\section{A. Dataset description}

During 50 cataract surgeries the view of the surgeon's microscope has been video recorded in a resolution of $1920 \times 1080$ pixels at 30 frames per second. The viewpoint is mostly static but the camera shakes and occasionally the zoom level is changed. On average, the surgery duration is 11 minutes so that over 9 hours of video are available. A total of 21 different surgical tools are used in the videos and up to 3 tools can be visible at a time. However, this is extremely rare $(0.04 \%)$ and most frames show no tools (45\%), one tool (38\%) or two tools $(17 \%)$. The videos have been independently annotated by two experts, which allows to ignore frames where the experts disagree during the evaluation. For each frame and surgical tool, a label indicates if the tool touches the eyeball. Note that this means a tool can be visible but still not annotated because it does not yet touch the eyeball. Also, multiple tools can be present in a single frame but bounding box information to distinguish the tools in the frame is not available. This is essentially a weakly supervised classification setting or more specifically multi-label classification. Finally, all 50 videos have been evenly split into a training and test set but labels are only provided for the training set.

\section{B. Dataset challenges and preprocessing}

The dataset poses some challenges that have to be addressed before being usable for training. First of all, the video resolution is very high at $1920 \times 1080$ pixels. This is a problem for CNNs as the required processing time and especially memory is directly influenced by the image resolution. However, the images cannot be scaled down arbitrarily because most tools are elongated objects of only about 30 pixels width. A resolution of $960 \times 540$ pixels was found to be a good compromise between resource demands and object size.

Due to the nature of video, subsequent frames are extremely similar to one another. They are heavily correlated and using neighboring frames during supervised learning yields almost no information gain. Therefore, only every sixth frame of each video is used during training which leads to $200 \mathrm{~ms}$ intervals between processed frames. No significant information is lost this way, but the required training time is reduced considerably.

Additionally, the similarity between neighboring frames has implications on the choice of validation data. Consider a random split of all frames into a large training and small validation set. It is highly likely that for each frame in the validation set either the predecessor or successor of that frame is part of the training set. The validation error would therefore be a significant underestimation of the test error. Instead, the training-validation-split is performed on the video level: 5 of the 25 training videos are set aside for validation purposes.

The dataset also exhibits a strong class imbalance. Because almost half of all frames do not show any tools, this subset of the data is undersampled to $40 \%$ of its original size. While the number of available training examples for each tool also varies considerably, the more important consideration is how many videos contain a sequence showing each tool. This is relevant because frames showing the same tool in different videos are a lot more varied than frames showing the same tool in a single video. In consequence, the available videos are distributed between training and validation set so that both contain some videos showing each tool. The distribution of the eventual split can be seen in Figure 4. Unfortunately, it is only possible to split the videos in ways that have some 


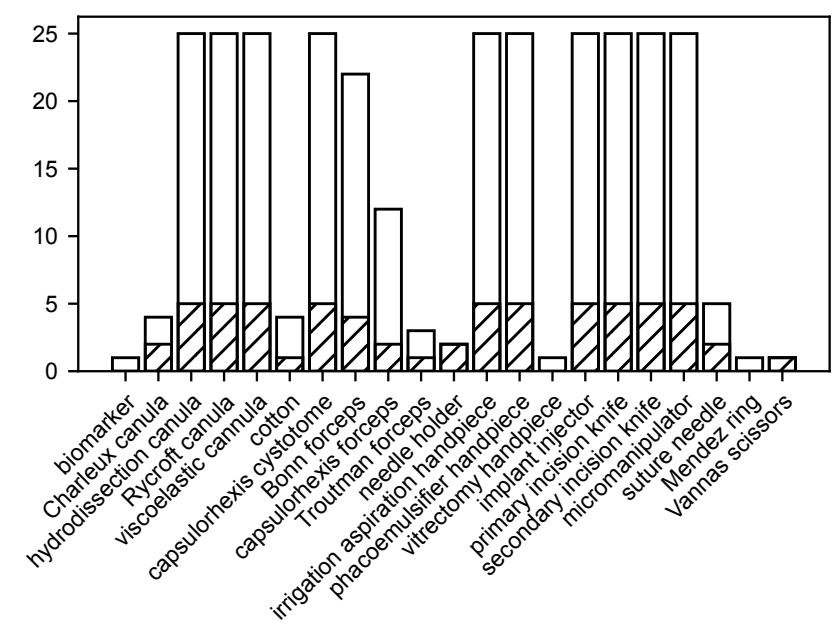

Fig. 4. Amount of videos showing each tool inside the training set (empty bars) and validation set (striped bars)

tools appearing only in the training or validation set. This necessitates to exclude the tools Vannas scissors, Mendez ring, vitrectomy handpiece, needle holder and biomarker from the experiments for validation purposes. Note that even for classes that appear in the same amount of videos, there are still great differences in the amount of available training examples. For example, the micromanipulator and implant injector are both present in every video but the former is used multiple times and for long periods per video while the latter is used exactly once for a few seconds per video.

The resulting training set contains about $43 \mathrm{k}$ images and 16 different classes. The validation set is comprised of all frames from the validation videos with a total of $73 \mathrm{k}$ images. The validation set is only larger than the training set because frames are skipped during training but not during validation.

\section{Data augmentation}

Because some tools only appear in a single training video the variability between the frames showing such tools is low. To tackle this problem, several image augmentation techniques are employed. First, the image is randomly cropped to $960 \times 540$ by scaling the original image down to $1024 \times 604$ and sampling horizontal and vertical offsets from a uniform distribution between 0 and 64. Afterwards, it is horizontally flipped in $50 \%$ of all cases. Then color augmentation as described by [23] is applied with $\alpha$ drawn from a standard normal distribution. Finally, the image is rotated around its center by a value chosen from a random uniform distribution between $-15^{\circ}$ and $15^{\circ}$.

\section{EXPERIMENTS}

\section{A. Training and evaluation procedure}

As usual for image data, pixel values are scaled to be in $[0,1]$ and then zero-centered by subtracting the mean pixel activations over the training set. In practice this involves calculating the average value for each pixel and color channel over the training set. The result is a "mean image" of the training set that can be subtracted from each training example.

All networks are trained with stochastic gradient descent and with 0.9 momentum. The learning rate starts at 0.05 in all cases and decays over the course of the training. The learning rate at each batch $n$ is computed as $0.05 /(1+d n)$ with decay factor $d$.

When training a FFE network the decay factor is $d_{\mathrm{FFE}}=$ $1 \mathrm{e}-3$, the batch size is 32 and the training is run for $20 \mathrm{k}$ iterations. When fine-tuning, the decay factor $d_{\mathrm{FT}}=1.25 \mathrm{e}-4$ is chosen, the batch size is 8 and the training is run for $60 \mathrm{k}$ iterations. The amount of training iterations was chosen in such a way that the validation AUC has already stagnated before finishing the training. In the fine-tuning case the batch size is that much smaller than in the FFE case because training all of ResNet's weights requires more memory than only training the few custom layers after a FFE ResNet. It should be noted that such a small batch size is not ideal when using batch normalization, because the batch statistics vary much more. However, the training of all networks still works well enough.

Experimental results are first obtained using the validation set as presented in Figure 4. Therefore, only 16 classes are distinguished instead of 21 . The assumption is that networks that perform best on the validation set will also perform best on the challenge's test set even though the networks will change slightly to accommodate the additional class outputs. For the final submission to the challenge, the best network is trained on all 25 training videos without a validation set. Training without a validation set is necessary because some tools are only present in a single video so that such a tool is either part of the training set or the validation set. This "blind" training procedure is the main motivation to use a static learning rate schedule because dynamic rules like reducing the learning rate when the AUC plateaus are not possible without a validation set.

The area under the ROC curve (AUC) is the performance metric used in all experiments. Each of the network's $c$ outputs is a score in $[0,1]$. A threshold is applied to decide whether that score means a tool is present or not. By varying this threshold the ROC curve is created. It is calculated separately for each of the $c$ outputs because it is designed for binary classification problems. Finally, the resulting values are averaged to get an aggregating performance metric for the whole network. This is sometimes called macro-average ROC.

\section{B. Results on validation set}

Comparison of transfer learning settings. The first series of experiments is performed to compare different transfer learning settings. Four instances of the FT and FFE family are trained using the standard classification head $a v g-f c$. The FT instances vary in the amount of ResNet layers that are frozen during training while the FFE instances vary in the amount of ResNet layers that are used as the feature extractor. The results are shown in Table I and Table II in column $a v g-f c$.

The FT instances perform consistently better than all FFE instances. This is plausible as the target dataset is very different 
TABLE I

EXPERIMENTAL RESULTS FOR FT NETWORKS

\begin{tabular}{|c|c|c|c|}
\hline \multicolumn{2}{|c|}{ Model configuration } & \multicolumn{2}{|c|}{ Val. AUC } \\
\hline Name & Frozen layers & $\overline{\mathrm{avg}-\mathrm{fc}}$ & conv-max \\
\hline FT0 & None & 0.9488 & 0.9225 \\
\hline FT22 & Partial (22) & 0.9522 & 0.9135 \\
\hline FT31 & Partial (31) & 0.9503 & 0.8909 \\
\hline FT40 & Partial (40) & 0.9440 & 0.8742 \\
\hline
\end{tabular}

TABLE II

EXPERIMENTAL RESULTS FOR FFE NETWORKS

\begin{tabular}{lllll}
\hline \multicolumn{3}{c}{ Model configuration } & \multicolumn{2}{c}{ Val. AUC } \\
Name & ResNet $k$ & Custom part & avg-fc & conv-max \\
\hline \hline FFE22 & 22 & Yes & 0.9073 & 0.8304 \\
FFE31 & 31 & Yes & 0.9285 & 0.9187 \\
FFE40 & 40 & Yes & 0.9365 & 0.9266 \\
FFE49 & 49 & Yes & $\mathbf{0 . 9 4 0 9}$ & 0.9180 \\
FFE49NC & 49 & No & 0.8161 & - \\
\hline
\end{tabular}

from ImageNet so that the more flexible fine-tuning instances should adapt better. Because the FT instances perform best, each of those four experiments is repeated a total of five times with random initializations and averaged to get more precise results. This allows to select the best instance for further experiments and will also allow to quantify improvements with greater certainty.

Among the FT instances a medium amount of frozen layers works best. When freezing either 22 or 31 layers of ResNet the results improve over freezing none or 40 layers. Both of these instances, FT22 and FT31, perform similarly well at 0.95 AUC though FT31 is cheaper to train because it has more frozen layers. Compared to FFE22 and FFE31, which have as many total ResNet layers as the FT22 and FT31 networks have frozen layers, fine-tuning performs better. There are two likely reasons for this: First, the trainable custom part appended to the FFE networks is of fixed, small capacity. In contrast, the trainable part of FT networks is the complete remainder of ResNet that has not been frozen. Therefore, more trainable capacity is available. Second, the weights in the FFE network's custom part are randomly initialized, while the FT network's weights benefit from improved initial conditions due to pretraining.

Among the FFE instances, the performance rises with the amount of ResNet layers that are used as the feature extractor. Interestingly, the experiment FFE49 which uses the full ResNet as a feature extractor achieves the best result with 0.94 AUC despite the dataset differences. However, this is solely due to the trainable custom layers inserted between the feature extractor and the classification head. In the experiment denoted as FFE49NC the trainable custom part is not included and the achieved performance drops significantly to 0.82 AUC.

To test how important the pretraining on ImageNet is, the completely trainable FT0 network is trained again starting
TABLE III

INFLUENCE OF PRETRAINING ON IMAGENET

\begin{tabular}{ccc}
\hline \multicolumn{2}{c}{ FT0 model } & Val. AUC \\
Pretrained & Iterations & avg-fc \\
\hline \hline Yes & $60 \mathrm{k}$ & $\mathbf{0 . 9 4 8 8}$ \\
No & $60 \mathrm{k}$ & 0.9180 \\
No & $100 \mathrm{k}$ & 0.9224 \\
\hline
\end{tabular}

from a random weights initialization. The results are shown in Table III When training for the same number of iterations as FT0 a significantly lower AUC of 0.92 is achieved. Even when training the network until the validation AUC stagnates (100k iterations), the final performance is still much lower than what is possible with pretraining. In conclusion, the pretraining is vital for good performance.

It is also of interest if the same performance can be achieved with lower resolution input images. Such a result would be very helpful as the training times and memory requirements would lower dramatically. The FT31 network is trained again with different configurations on $480 \times 270$ pixel input images but no AUC better than 0.93 could be achieved. Consequently, the resolution is not lowered.

Comparison of classification heads. A second series of experiments is conducted to compare between the two possible classification head types: standard global average-pooling followed by a fully connected layer (avg-fc) and the proposal for weakly supervised learning by Oquab et al. [9] using a fully convolutional network and a global max-pooling layer (conv-max). The previous FT and FFE experiments are repeated with the conv-max classification head and the results are shown in Table \ and Table П in column conv-max.

The conv-max classification head performs consistently worse than the standard $a v g-f c$ approach in FT and FFE settings. Oquab et al. [9] report good performance on PASCAL VOC 2012 using their approach, which is specifically motivated by the multi-label, no bounding-box nature of their dataset. Since the CATARACTS dataset shares these characteristics, the approach should be well suited but unexpectedly fails. A possible explanation lies in the difference between the CATARACTS and PASCAL VOC 2012 datasets. The average amount of classes per image is 1.0 for CATARACTS (after applying the preprocessing steps) and 2.4 for PASCAL VOC 2012. Furthermore, the CATARACTS dataset contains at least some frames for each tool that show no other tools. To test if these circumstances are responsible for the bad performance, the training on CATARACTS is repeated only with frames that show at least two tools, bringing the classes per image up to 2.0. Unfortunately, this reduces the dataset in size a lot so that good results cannot be expected. The FT31 network is trained again on the modified dataset-once with each classification head. The $a v g-f c$ variant achieves 0.60 AUC, while the convmax variant does not even learn properly and gets stuck on 0.50 AUC. Since $a v g-f c$ still performs better the assumption cannot be validated. 
TABLE IV

EXPERIMENTAL RESULTS WITH A WEIGHTED LOSS FUNCTION

\begin{tabular}{lllc}
\hline \multicolumn{3}{c}{ Model configuration } & Val. AUC \\
Name & Weighted & Iterations & $\overline{\text { avg-fc }}$ \\
\hline \hline FT22 & No & $60 \mathrm{k}$ & 0.9522 \\
FT22* & Yes & $25 \mathrm{k}$ & 0.9552 \\
FT31 & No & $60 \mathrm{k}$ & 0.9503 \\
FT31* & Yes & $25 \mathrm{k}$ & $\mathbf{0 . 9 5 8 1}$ \\
\hline
\end{tabular}

Weighted loss function. Due to the heavy class imbalance in the dataset, minority classes might not be trained as well as majority classes, which dominate the gradient direction. To verify if better results can be achieved when actively dealing with the class imbalance, a weighted loss function is employed. To this end, each class is associated with a weight

$$
w_{i}=\sqrt{\frac{\max \left\{f_{j} \mid 1 \leq j \leq c\right\}}{f_{i}}},
$$

where $f_{i}$ is the frequency with that class $i$ appears in the dataset. Ignoring the square root for a moment, this associates a weight of $x$ to each class that appears $1 / x$ as many times as the majority class. Because the frequency differences in this dataset are quite drastic, the square root was applied to prevent huge weights and therefore gradient descent steps. Before the backwards pass, the cross-entropy loss associated with each output (class) $i$ is multiplied by the class weight $w_{i}$. This leads to all weight updates during backpropagation being scaled by the same factor.

The best FT instances, FT22 and FT31, are trained again five times with the described weighted loss and the averaged results are shown in Table IV.

During experiments with the weighted loss, overfitting is immediately observed. A plausible explanation is that all class weights $w_{i}$ are greater or equal to one, which leads to larger gradient descent steps in general. Therefore, the training may progress faster and overfitting is observed earlier. Training the same networks for $25 \mathrm{k}$ iterations instead of $60 \mathrm{k}$ leads prevents the overfitting. In the end, the weighted loss improves the AUC for both networks slightly and FT31* achieves the highest overall performance of 0.96 AUC.

Regularization. Because the previous experiments overfit quickly, regularization might help. The networks are trained again with $\mathrm{L} 2$ regularization of 1e-4 and 1e-5 on all weights and biases. However, the performance stays similar or degrades so no further experiments in this direction are conducted.

Final network. For clarity, a complete description of the best performing network, FT31*, follows. The network consists of the first 49 layers of ResNet followed by the avg-fc classification head. The first 31 convolutional layers of ResNet are frozen so that their weights are not modified during training. The gradient of each output node is scaled by the associated class weight as described previously. The network is pretrained on ImageNet and then fine-tuned for $25 \mathrm{k}$ iterations
TABLE V

RESULTS OF FT31* ON THE TEST SET

\begin{tabular}{lll}
\hline Tool & Test set AUC \\
\hline \hline Average & 0.9568 & \\
\hline secondary incision knife & 0.9978 & \\
capsulorhexis cystotome & 0.9976 & \\
phacoemulsifier handpiece & 0.9971 & \\
vitrectomy handpiece & 0.9932 & \\
irrigation/aspiration handpiece & 0.9913 & \\
micromanipulator & 0.9912 & \\
Rycroft canula & 0.9908 & \\
capsulorhexis forceps & 0.9888 & \\
primary incision knife & 0.9848 & \\
Mendez ring & 0.9814 & \\
suture needle & 0.9796 & \\
cotton & 0.9759 & \\
Bonn forceps & 0.9726 & \\
hydrodissection canula & 0.9717 & \\
needle holder & 0.9709 & \\
Vannas scissors & 0.9673 & \\
Troutman forceps & 0.9656 & \\
implant injector & 0.9644 & \\
viscoelastic cannula & 0.9545 & \\
Charleux canula & 0.8771 & \\
biomarker & 0.5797 & $\mathbf{}$ \\
\hline
\end{tabular}

on CATARACTS. Other settings such as learning rate or batch size follow the description in Section $\mathrm{V}-\mathrm{A}$

\section{Results on test set}

The best network found in all experiments on the validation set, FT31*, is used to classify the challenge's test set. The average AUC over all classes is 0.957 and a detailed evaluation broken down by class is shown in Table $\mathrm{V}$. The test set AUCs by tool are somewhat correlated to each tool's training subset size. For the three classes irrigation/aspiration handpiece, phacoemulsifier handpiece and micromanipulator the network achieves more than $0.99 \mathrm{AUC}$ and these are also the classes that have the most training examples. In contrast, the biomarker detection is hardly better than chance and it is one of the classes that appear in only a single training video. Additionally, the biomarker appears in the fewest video frames in total. The combination of not having enough data and simultaneously being assigned the largest class weight probably leads to severe overfitting. It should still be noted that some classes such as the Mendez ring perform well despite having few training examples. In this case the likely reason is that the Mendez ring looks very different from all other tools and is therefore easy to distinguish.

\section{CONCLUSION}

A surgical tool detection network based on the 50-layer Residual Network has been trained on the CATARACTS challenge dataset. Images extracted from the training videos 
are highly correlated and each image is assigned a set of labels instead of exactly one label. Furthermore, there is an extreme class imbalance. In consequence, the dataset is heavily preprocessed and augmented. The class imbalance in combination with the specific distribution of tool use over the videos made it necessary to validate on a subset of the data that contains only 16 instead of all 21 classes. Even worse, it makes it necessary to blindly train the final network without a validation set.

Different transfer learning settings are compared in a series of experiments. Fine-tuning ResNet achieves consistently better results than using ResNet as a fixed feature extractor in combination with a custom classifier. Next, a specialized classification head to deal with the multi-label nature of the problem is tested but does not prove useful. However, using a weighted loss function that increases the loss for underrepresented classes slightly improves the model's performance.

The resulting network works exceptionally well for some tools but performance suffers in other cases because not enough training data is available. Consequently, with a bigger dataset it should be possible to create a classifier with close to perfect performance. Another possibility to improve performance is creating an ensemble of networks starting from different random initializations or even with different architectures. This might be especially helpful for the biomarker, as the presumed reason for the bad detection performance is overfitting.

\section{REFERENCES}

[1] Z. Zhou, J. Shin, L. Zhang, S. Gurudu, M. Gotway, and J. Liang, "Finetuning convolutional neural networks for biomedical image analysis: Actively and incrementally," in The IEEE Conference on Computer Vision and Pattern Recognition (CVPR), July 2017.

[2] X. Wang, Y. Peng, L. Lu, Z. Lu, M. Bagheri, and R. M. Summers, "Chestx-ray8: Hospital-scale chest X-ray database and benchmarks on weakly-supervised classification and localization of common thorax diseases," in The IEEE Conference on Computer Vision and Pattern Recognition (CVPR), July 2017.

[3] H. A. Hajj, G. Quellec, M. Lamard, G. Cazuguel, and B. Cochener, "Coarse-to-fine surgical instrument detection for cataract surgery monitoring," CoRR, vol. abs/1609.05619, 2016. [Online]. Available: http://arxiv.org/abs/1609.05619

[4] K. Charrière, G. Quellec, M. Lamard, D. Martiano, G. Cazuguel, G. Coatrieux, and B. Cochener, "Real-time analysis of cataract surgery videos using statistical models," Multimedia Tools and Applications, vol. 76, no. 21, pp. 22 473-22 491, Nov 2017. [Online]. Available: https://doi.org/10.1007/s11042-017-4793-8

[5] D. Bouget, M. Allan, D. Stoyanov, and P. Jannin, "Vision-based and marker-less surgical tool detection and tracking: A review of the literature," Medical Image Analysis, vol. 35, pp. 633-654, Jan. 2017 [Online]. Available: http://dx.doi.org/10.1016/j.media.2016.09.003

[6] K. He, X. Zhang, S. Ren, and J. Sun, "Deep residual learning for image recognition," in 2016 IEEE Conference on Computer Vision and Pattern Recognition (CVPR), June 2016, pp. 770-778.

[7] O. Russakovsky, J. Deng, H. Su, J. Krause, S. Satheesh, S. Ma, Z. Huang, A. Karpathy, A. Khosla, M. Bernstein, A. C. Berg, and L. Fei-Fei, "ImageNet Large Scale Visual Recognition Challenge," International Journal of Computer Vision (IJCV), vol. 115, no. 3, pp. 211-252, 2015

[8] A. Karpathy, G. Toderici, S. Shetty, T. Leung, R. Sukthankar, and L. Fei-Fei, "Large-scale video classification with convolutional neural networks," in Proceedings of the 2014 IEEE Conference on Computer Vision and Pattern Recognition, ser. CVPR '14. Washington, DC, USA: IEEE Computer Society, 2014, pp. 1725-1732. [Online]. Available: http://dx.doi.org/10.1109/CVPR.2014.223
[9] M. Oquab, L. Bottou, I. Laptev, and J. Sivic, "Is object localization for free? Weakly-supervised learning with convolutional neural networks," in 2015 IEEE Conference on Computer Vision and Pattern Recognition (CVPR), June 2015, pp. 685-694.

[10] J. Huang, V. Rathod, C. Sun, M. Zhu, A. Korattikara, A. Fathi, I. Fischer, Z. Wojna, Y. Song, S. Guadarrama, and K. Murphy, "Speed/accuracy trade-offs for modern convolutional object detectors," CoRR, vol. abs/1611.10012, 2016. [Online]. Available: http://arxiv.org/ abs/1611.10012

[11] Y. Gong, Y. Jia, T. Leung, A. Toshev, and S. Ioffe, "Deep convolutional ranking for multilabel image annotation," CoRR, vol. abs/1312.4894, 2013. [Online]. Available: http://arxiv.org/abs/1312.4894

[12] Y. Wei, W. Xia, M. Lin, J. Huang, B. Ni, J. Dong, Y. Zhao, and S. Yan, "HCP: A flexible CNN framework for multi-label image classification," IEEE Transactions on Pattern Analysis and Machine Intelligence, vol. 38, no. 9, pp. 1901-1907, Sept 2016.

[13] M. L. Zhang and Z. H. Zhou, "A review on multi-label learning algorithms," IEEE Transactions on Knowledge and Data Engineering, vol. 26, no. 8, pp. 1819-1837, Aug 2014.

[14] J. Yosinski, J. Clune, Y. Bengio, and H. Lipson, "How transferable are features in deep neural networks?" in Proceedings of the 27th International Conference on Neural Information Processing Systems, ser NIPS'14. Cambridge, MA, USA: MIT Press, 2014, pp. 3320-3328. [Online]. Available: http://dl.acm.org/citation.cfm?id=2969033.2969197

[15] T. Fawcett, "An introduction to ROC analysis," Pattern Recognition Letters, vol. 27, no. 8, pp. 861 - 874, 2006. [Online]. Available: http://www.sciencedirect.com/science/article/pii/S016786550500303X

[16] Y. Sun, A. Wong, and M. S. Kamel, "Classification of imbalanced data: A review," vol. 23, no. 4, 112009.

[17] M. A. Mazurowski, P. A. Habas, J. M. Zurada, J. Y. Lo, J. A. Baker, and G. D. Tourassi, "Training neural network classifiers for medical decision making: The effects of imbalanced datasets on classification performance," Neural Networks, vol. 21, no. 2, pp. 427 - 436, 2008. [Online]. Available: http://www.sciencedirect.com/science/article/pii/S0893608007002407

[18] G. Quellec, M. Lamard, B. Cochener, and G. Cazuguel, "Real-time segmentation and recognition of surgical tasks in cataract surgery videos," IEEE Transactions on Medical Imaging, vol. 33, no. 12, pp. 2352-2360, Dec 2014.

[19] G. Quellec, M. Lamard, B. Cochener, and G. Cazuguel, "Real-time task recognition in cataract surgery videos using adaptive spatiotemporal polynomials," IEEE Transactions on Medical Imaging, vol. 34, no. 4, pp. 877-887, Apr. 2015.

[20] A. P. Twinanda, S. Shehata, D. Mutter, J. Marescaux, M. de Mathelin, and N. Padoy, "EndoNet: A deep architecture for recognition tasks on laparoscopic videos," CoRR, vol. abs/1602.03012, 2016. [Online]. Available: http://arxiv.org/abs/1602.03012

[21] C. Szegedy, W. Liu, Y. Jia, P. Sermanet, S. Reed, D. Anguelov, D. Erhan, V. Vanhoucke, and A. Rabinovich, "Going deeper with convolutions," in 2015 IEEE Conference on Computer Vision and Pattern Recognition (CVPR), June 2015, pp. 1-9.

[22] S. Ioffe and C. Szegedy, "Batch normalization: Accelerating deep network training by reducing internal covariate shift," in Proceedings of the $32 N d$ International Conference on International Conference on Machine Learning - Volume 37, ser. ICML'15. JMLR.org, 2015, pp. 448-456. [Online]. Available: http://dl.acm.org/citation.cfm?id=3045118.3045167

[23] A. Krizhevsky, I. Sutskever, and G. E. Hinton, "ImageNet classification with deep convolutional neural networks," in Proceedings of the 25th International Conference on Neural Information Processing Systems, ser. NIPS'12. USA: Curran Associates Inc., 2012, pp. 1097-1105. [Online] Available: http://dl.acm.org/citation.cfm?id=2999134.2999257 